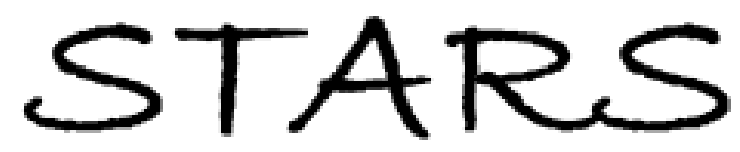

University of Central Florida

STARS

$1-1-2013$

\title{
Generation of high-flux attosecond extreme ultraviolet continuum with a 10 TW laser
}

\author{
Y. Wu \\ University of Central Florida \\ E. Cunningham \\ University of Central Florida
}

H. Zhang

University of Central Florida

J. Li

University of Central Florida

M. Chini

University of Central Florida

Find similar works at: https://stars.library.ucf.edu/facultybib2010

University of Central Florida Libraries http://library.ucf.edu

See next page for additional authors

This Article is brought to you for free and open access by the Faculty Bibliography at STARS. It has been accepted for inclusion in Faculty Bibliography 2010 s by an authorized administrator of STARS. For more information, please contactSTARS@ucf.edu.

\section{Recommended Citation}

Wu, Y.; Cunningham, E.; Zhang, H.; Li, J.; Chini, M.; Wang, X.; Wang, Y.; Zhao, K.; and Chang, Z., "Generation of high-flux attosecond extreme ultraviolet continuum with a 10 TW laser" (2013). Faculty Bibliography 2010s. 4868.

https://stars.library.ucf.edu/facultybib2010/4868

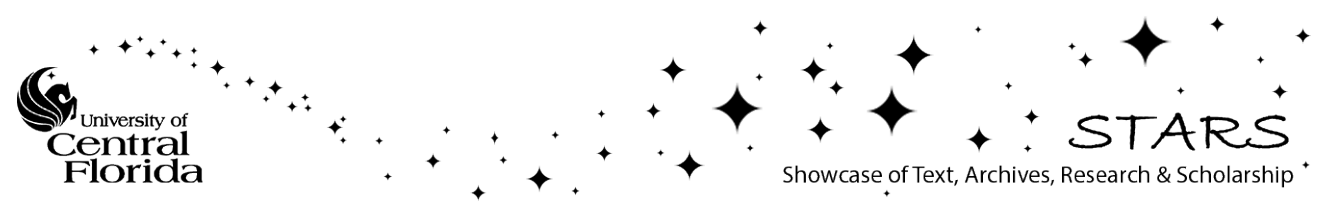


Authors

Y. Wu, E. Cunningham, H. Zhang, J. Li, M. Chini, X. Wang, Y. Wang, K. Zhao, and Z. Chang 


\section{Generation of high-flux attosecond extreme ultraviolet continuum with a $10 \mathrm{TW}$ laser}

Cite as: Appl. Phys. Lett. 102, 201104 (2013); https://doi.org/10.1063/1.4807395

Submitted: 19 March 2013. Accepted: 04 May 2013 . Published Online: 21 May 2013

Y. Wu, E. Cunningham, H. Zang, J. Li, M. Chini, X. Wang, Y. Wang, K. Zhao, and Z. Chang
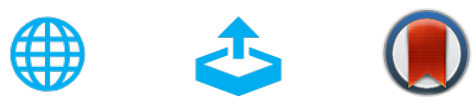

View Online

Export Citation

\section{ARTICLES YOU MAY BE INTERESTED IN}

Polarization gating of high harmonic generation in the water window

Applied Physics Letters 108, 231102 (2016); https://doi.org/10.1063/1.4953402

\section{A high-flux high-order harmonic source}

Review of Scientific Instruments 84, 073103 (2013); https://doi.org/10.1063/1.4812266

Generation of high energy $10 \mathrm{fs}$ pulses by a new pulse compression technique

Applied Physics Letters 68, 2793 (1996); https://doi.org/10.1063/1.116609

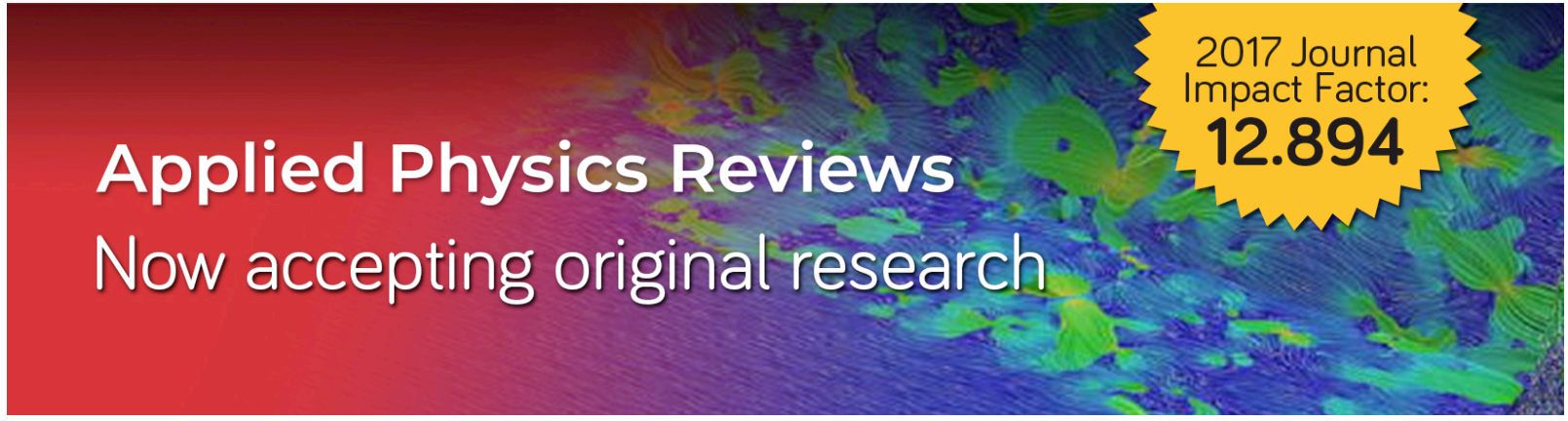




\title{
Generation of high-flux attosecond extreme ultraviolet continuum with a 10 TW laser
}

\author{
Y. Wu,${ }^{1}$ E. Cunningham, ${ }^{1}$ H. Zang,${ }^{1,2}$ J. Li, ${ }^{1}$ M. Chini, ${ }^{1}$ X. Wang, ${ }^{1,3}$ Y. Wang, ${ }^{1}$ K. Zhao, ${ }^{1}$ \\ and Z. Chang ${ }^{1, a)}$ \\ ${ }^{1}$ CREOL and Department of Physics, University of Central Florida, Orlando, Florida 32816, USA \\ ${ }^{2}$ Institute of Atomic and Molecular Physics, Sichuan University, Chengdu 610054, China \\ ${ }^{3}$ Department of Physics, National University of Defense Technology, Hunan 410073, China
}

(Received 19 March 2013; accepted 4 May 2013; published online 21 May 2013)

\begin{abstract}
We report a laser system that delivers $15 \mathrm{fs}$ pulses with $200 \mathrm{~mJ}$ energy at a $10 \mathrm{~Hz}$ repetition rate. The broadband spectrum extending from $700 \mathrm{~nm}$ to $900 \mathrm{~nm}$ was obtained by seeding a two-stage Ti:sapphire chirped-pulse power amplifier with sub-mJ white-light pulses from a gas-filled hollow-core fiber. With this laser, an extreme ultraviolet (XUV) super-continuum supporting 230 as isolated attosecond pulses at $35 \mathrm{eV}$ was generated using the generalized double optical gating technique. The XUV pulse energy was $\sim 100 \mathrm{~nJ}$ at the exit of the argon gas target. (C) 2013 AIP Publishing LLC. [http://dx.doi.org/10.1063/1.4807395]
\end{abstract}

Time-resolved experiments with attosecond resolution have been performed thus far by combining weak extreme ultraviolet (XUV) attosecond pulses with stronger near infrared (NIR) femtosecond lasers, which has limited the scope of attosecond science to the study of laser-induced strong-field processes. Isolated attosecond pulses with high photon flux are required for conducting true attosecond pump-attosecond probe experiments to study electron dynamics in atoms, molecules, and condensed matter. ${ }^{1}$ At current, significant progress has been made in developing "gating" schemes capable of isolating a single attosecond pulse from high harmonic generation $(\mathrm{HHG}),{ }^{2}$ but these XUV pulses are still restricted to energies less than $10 \mathrm{~nJ}^{3}{ }^{3}$ This is because most generation schemes require few-cycle driving NIR pulses that are similarly limited in their energies to $\sim 1 \mathrm{~mJ}$.

In order to scale the isolated attosecond pulse energy to the microjoule level, driving lasers with higher power are required. While the option of using optical parametric chirped pulse amplification (OPCPA) offers the advantage of few-cycle laser pulses with scalable energy, the specialized pump laser technology for OPCPA is still underdeveloped. On the other hand, widely available Ti:Sapphire-based chirped-pulse amplification (CPA) laser systems are capable of achieving $\sim 100$ TW peak power, but their output pulses-typically lasting 30 fs or longer-contain too many optical cycles to be used with even the least-restrictive gating schemes.

In this letter, we report the generation of $100 \mathrm{~nJ}$ isolated attosecond pulses from a unique high-energy, short-pulse laser system employing Generalized Double Optical Gating (GDOG), which is a combination of polarization gating and two-color gating. ${ }^{4}$ In GDOG, the laser field is manipulated to be linearly polarized only within a single-optical-cycle "gate" at the center of the pulse, whereas the leading and trailing edges are elliptically polarized. A linearly polarized second-harmonic field is added to break the field symmetry, restricting attosecond pulse generation to a single event

a)Zenghu.Chang@ucf.edu within the gate. While the GDOG method can be applied to both few and multi-cycle lasers, the driving laser pulse duration remains a key parameter, as only the energy contained in the linearly polarized cycle of the laser contributes to the generation of the isolated attosecond pulse. If the pulse duration is too long, the leading cycles of the pulse pre-ionize many of the available atoms before the arrival of the linear gated cycle, thus reducing the conversion efficiency. This effect can be mitigated by keeping the duration of the driving laser pulse as short as possible. To this end, we developed a Ti:sapphire laser system optimized to provide both highenergy $(200 \mathrm{~mJ})$ and short-duration (15 fs) pulses for implementing GDOG.

The optical layout of the driving laser and the setup for generating isolated attosecond pulses is shown schematically in Fig. 1. The main challenge of reaching 15 fs above the $10 \mathrm{TW}$ level is the suppression of spectral gain narrowing. This was resolved by distributing the high gain of the system $\left(\sim 10^{9}\right)$ over two chirped-pulse amplifiers. The significant spectral narrowing in the first CPA, which has a total gain of $10^{6}$, is recovered by spectral broadening in a neon gas-filled hollow-core fiber. The resulting millijoule-level white-light pulses are then used to seed the second CPA. Even though the pulses proceed to reach energies of several hundred millijoule, the final spectrum is spared from severe gain narrowing because the pulses only experience a total gain of $10^{3}$-orders of magnitude smaller than what is typical of other $10 \mathrm{TW}$-class lasers.

Spectra from various stages of the laser are shown in Fig. 2. The first CPA consists of a two-stage multi-pass Ti:sapphire amplifier seeded by a broadband Ti:sapphire oscillator. The output pulses $(1 \mathrm{kHz}, 20 \mathrm{fs}, 1.5 \mathrm{~mJ})$ are launched into a neon-filled hollow-core fiber $(1.5 \mathrm{~m}$ length, $450 \mu \mathrm{m}$ core diameter, 1.4 bar gas pressure) to generate a white-light continuum with spectrum from $550 \mathrm{~nm}$ to $950 \mathrm{~nm}$. The center mode of the spectrally broadened pulses is used to seed the second CPA. ${ }^{5}$

The sub-mJ broadband seed pulses first pass through an Offner-type stretcher, ${ }^{6}$ whose mirror curvatures are 


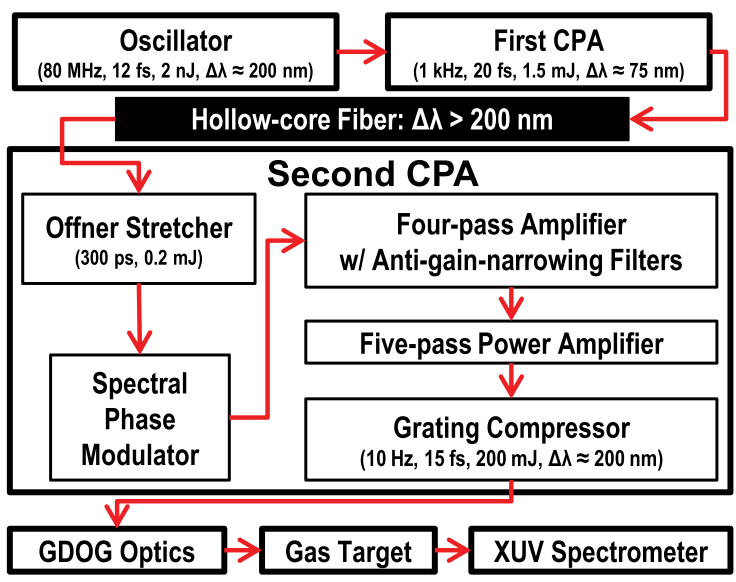

FIG. 1. Block diagram representing the $10 \mathrm{~Hz}, 10 \mathrm{TW}$, Ti:sapphire-based laser system with accompanying HHG and measurement setup.

optimized from $680 \mathrm{~nm}$ to $900 \mathrm{~nm}$ to minimize spatial chirp. The $300 \mathrm{ps}, 0.2 \mathrm{~mJ}$ positively chirped pulses are then amplified to $30 \mathrm{~mJ}$ in a four-pass amplifier operating at $10 \mathrm{~Hz}$. This Brewster-cut Ti:sapphire crystal is pumped from both sides by relay-imaged portions of a Q-switched frequencydoubled Nd:YAG laser (Quanta Ray Pro 350). Specially designed filters with a transmission minimum near $780 \mathrm{~nm}$ are inserted into the first three passes to alleviate gainnarrowing effects in this stage. ${ }^{7}$

The second stage power amplifier employs a large-area, flat-cut Ti:sapphire crystal, which is pumped on one side by the remainder of the first-stage pump laser and on the other side by the full energy of another Nd:YAG laser (Quanta Ray Pro 350) with high $\mathrm{M}^{2}$ factor. Two diffractive optical

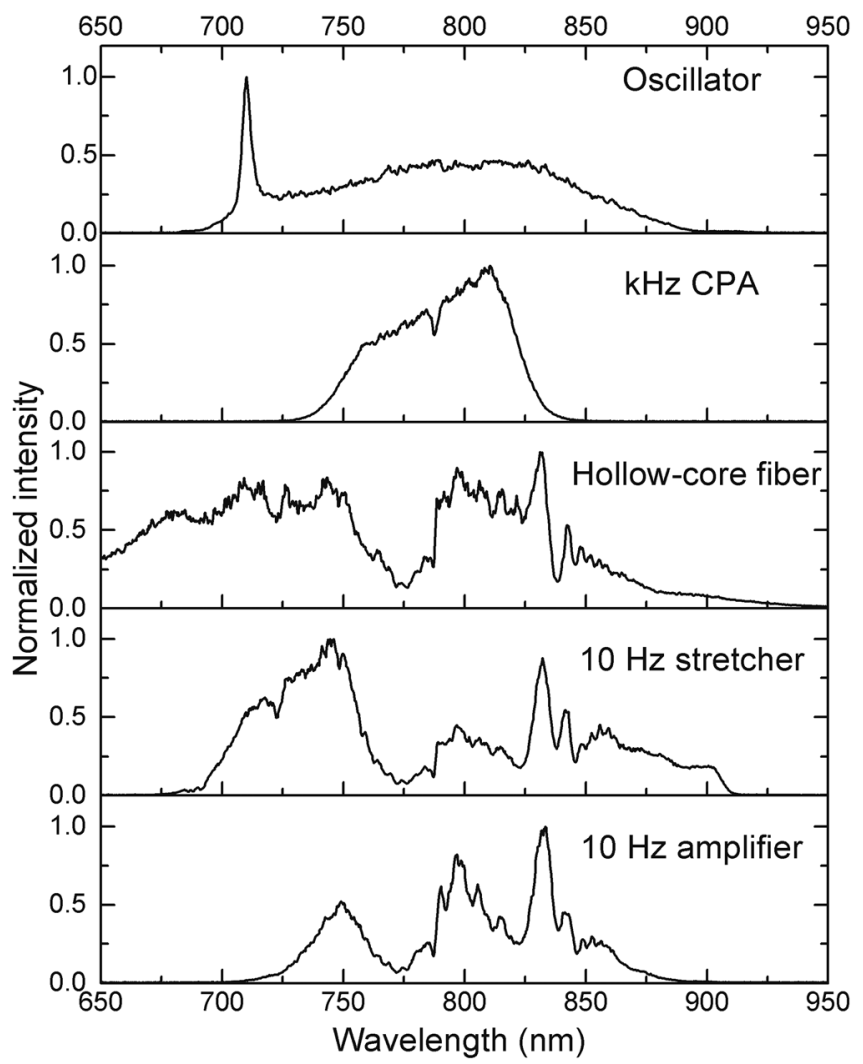

FIG. 2. Spectra taken at successive stages of the laser system. homogenizers regulate the profiles of the pump beams, ensuring that no "hot" structures are generated in the NIR pulse during amplification. ${ }^{8}$ Both stages are water-cooled to room temperature, while divergent lenses are used to compensate the thermal lens. The energy of the amplified pulse reaches up to $700 \mathrm{~mJ}$. The spectrum of these pulses extends from $700 \mathrm{~nm}$ to $900 \mathrm{~nm}$, which supports 12.2 fs transformlimited pulses. The long term stability, RMS/mean, is $1.6 \%$, whereas the shot-to-shot fluctuation is $\pm 4.4 \%$ for $99 \%$ of all laser pulses over $6 \mathrm{~h}$.

The beam is expanded to a diameter of $35 \mathrm{~mm}$ before it is sent under vacuum to a pulse compressor, which features a pair of $1400 \mathrm{l} / \mathrm{mm}$ gratings and an overall throughput of $50 \%$. The pulse compression was characterized by a singleshot second-harmonic FROG. First, the separation and incident angle of the gratings were fine-tuned manually to obtain a spectral phase that changed only gradually over any narrow range. This was done so that the high-order phase distortions could be compensated by a low-loss, 4 -zero-dispersion adaptive phase modulator, ${ }^{9}$ which is located between the stretcher and the first amplification stage. In the phase modulator, the laser beam is angularly dispersed by a Brewster-cut SF10 prism and re-collimated onto $50 \mathrm{~mm}$-long, 20-channel linear piezoelectric deformable mirror (OKO Technologies) using a dielectric mirror with a focal length of $1.5 \mathrm{~m}$. The overall transmission of this phase modulator reaches $85 \%$ for a bandwidth over $200 \mathrm{~nm}$.

For optimizing the pulse compression, the FROG traces were used as the fitness function for an evolutionary algorithm, which controlled the voltages applied to the deformable mirror actuators. After running the algorithm to convergence (usually $\sim 20$ iterations), a pulse duration of $15.1 \mathrm{fs}$ could be obtained, as displayed in Fig. 3. The energy of the pulse was $200 \mathrm{~mJ}$ for a $400 \mathrm{~mJ}$ input, which indicates that the peak power of the laser reached $13 \mathrm{TW}$. Even higher power $(30 \mathrm{TW})$ is expected once the current gratings are replaced with a larger-dimension, higher-throughput pair capable of accommodating the $700 \mathrm{~mJ}$ input. The carrierenvelope phase (CEP) of the laser is not stabilized due to two major challenges for CEP locking. First, the maximum speed of f-to-2f CEP measurement is limited to the laser repetition rate, which makes it difficult to compensate phase variations faster than $10 \mathrm{~Hz}$. Second, the large shot-to-shot fluctuation of the laser energy leads to significant error in phase measurement.

For isolated attosecond pulse generation, the laser intensity in the gas target must be kept below the ionization saturation intensity, which is $<10^{15} \mathrm{~W} / \mathrm{cm}^{2}$ for argon. Therefore, the high-power laser beam is loosely focused with a $6.5 \mathrm{~m}$-focal length mirror. Figure 4 shows the focal spot profile with a line-out in both the vertical and horizontal direction. The diameter of the focal spot is $0.31 \mathrm{~mm}$ (FWHM). The corresponding long confocal length allows the usage of a long gas cell with low gas density, which showed high conversion efficiency in producing coherent femtosecond XUV pulses with HHG. ${ }^{10}$

The laser field for GDOG is obtained by transforming the $15 \mathrm{fs}$ linearly polarized field with a set of birefringent optics. ${ }^{4}$ To achieve a gate width less than one optical cycle, the laser pulses are sent through a $1.070 \mathrm{~mm}$ quartz plate, 

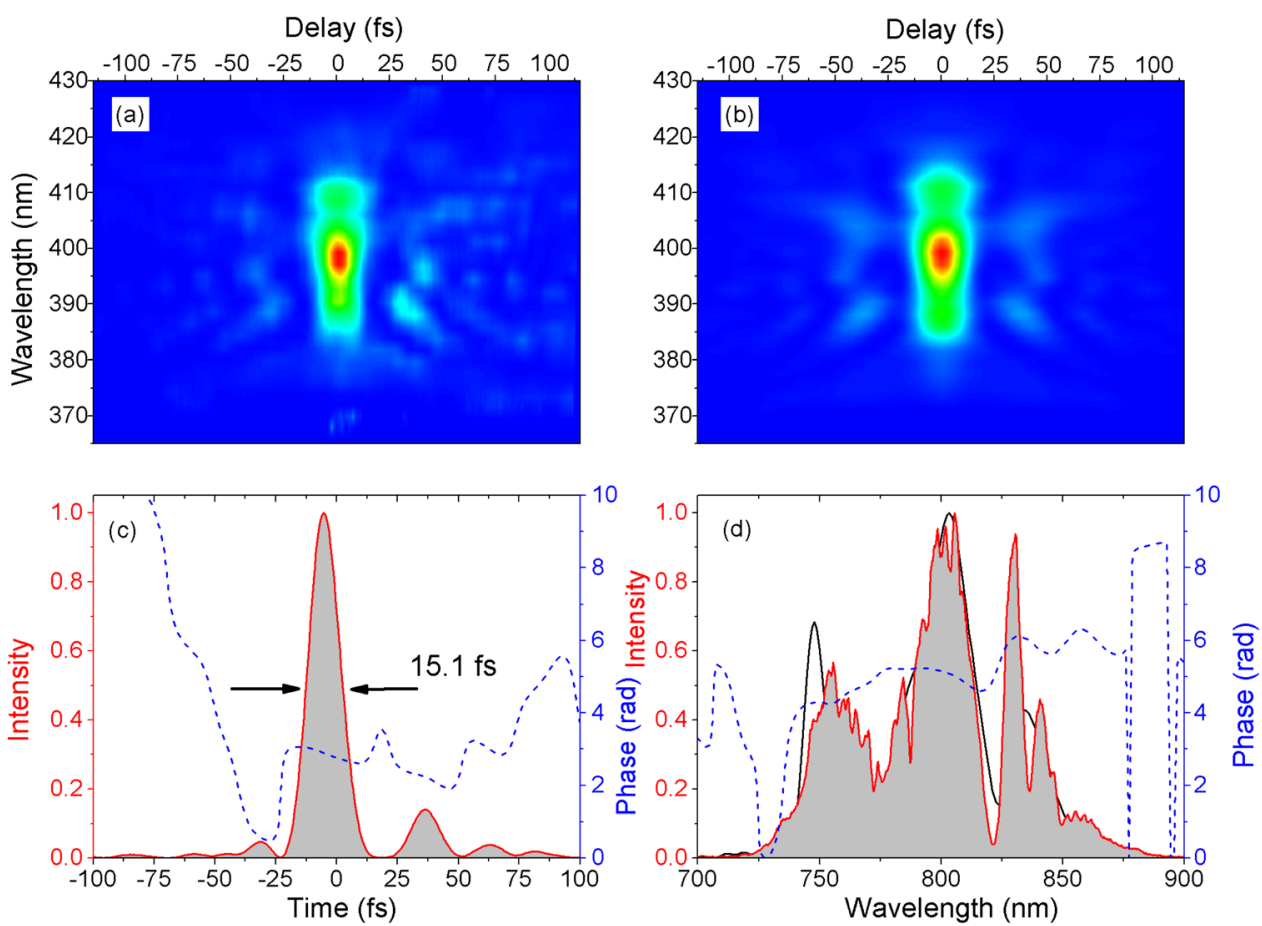

FIG. 3. Characterization of the $15.1 \mathrm{fs}$ laser pulse by SHG FROG. (a) The measured FROG trace. (b) The reconstructed FROG trace. (c) The retrieved pulse shape (solid line) and phase (dashed line). (d) The retrieved power spectrum (solid back line) and phase (dashed line) with the independently measured spectrum (solid red line). three $0.3 \mathrm{~mm}$ BK7 Brewster windows, a $0.440 \mathrm{~mm}$ quartz plate, and a $0.141 \mathrm{~mm}$ barium borate crystal (BBO) located directly after the focusing mirror. After the GDOG optics, the focused laser beam enters a $100 \mathrm{~mm}$-long gas cell, where attosecond pulses were generated in argon held at 4 Torr. Both ends of the cell were sealed with glass windows, each having $1 \mathrm{~mm}$-diameter holes through which the NIR beam and XUV beam can pass. To eliminate the residual driving laser that propagates collinearly with the XUV light, the output beam from the gas cell was filtered by two silicon mirrors set at the Brewster angle of the NIR laser. ${ }^{11}$

The XUV spectra under various gating conditions were measured with a home-built high-resolution XUV grating spectrometer. ${ }^{12}$ Figure 5 shows single-shot HHG spectra of argon while using no gating $(800 \mathrm{~nm}$ linearly polarized pulse), two-color gating (a second-harmonic field added to the linear fundamental field), and GDOG. As expected, wellresolved odd harmonics were generated when no gating was applied, and both odd and even harmonics appeared with the two-color laser field. These peaks merged to a continuum for every laser shot under the GDOG field. The profile of the XUV spectrum generated with GDOG changed from shot to shot, and we are working on determining the correlation between the spectrum shape and the CEP by tagging the phase value with the single shot XUV spectrum. If the dispersion of the XUV spectrum were to be compensated to the Fourier-transform limit by an appropriate method, ${ }^{13}$ the corresponding pulse duration would be 230 as.

The energy of the XUV pulse was measured to be $0.5 \mathrm{~nJ}$ with an XUV photodiode (Opto Diode Corp.) placed behind two $300 \mathrm{~nm}$-thick aluminum filters. This corresponds to an estimated XUV energy generated at the exit of the gas cell of $100 \mathrm{~nJ}$, assuming a $50 \%$ reflectance off each of the two silicon mirrors and a $2 \%$ total transmission through the two filters with $20 \mathrm{~nm}$ oxidation layers.

In conclusion, we have developed a 15 fs, 13 TW high-power laser system with a $10 \mathrm{~Hz}$ repetition rate for generating high-flux isolated attosecond pulses. This system represents the highest extent to which white-light from a hollow-core fiber has been amplified, allowing the pulses to
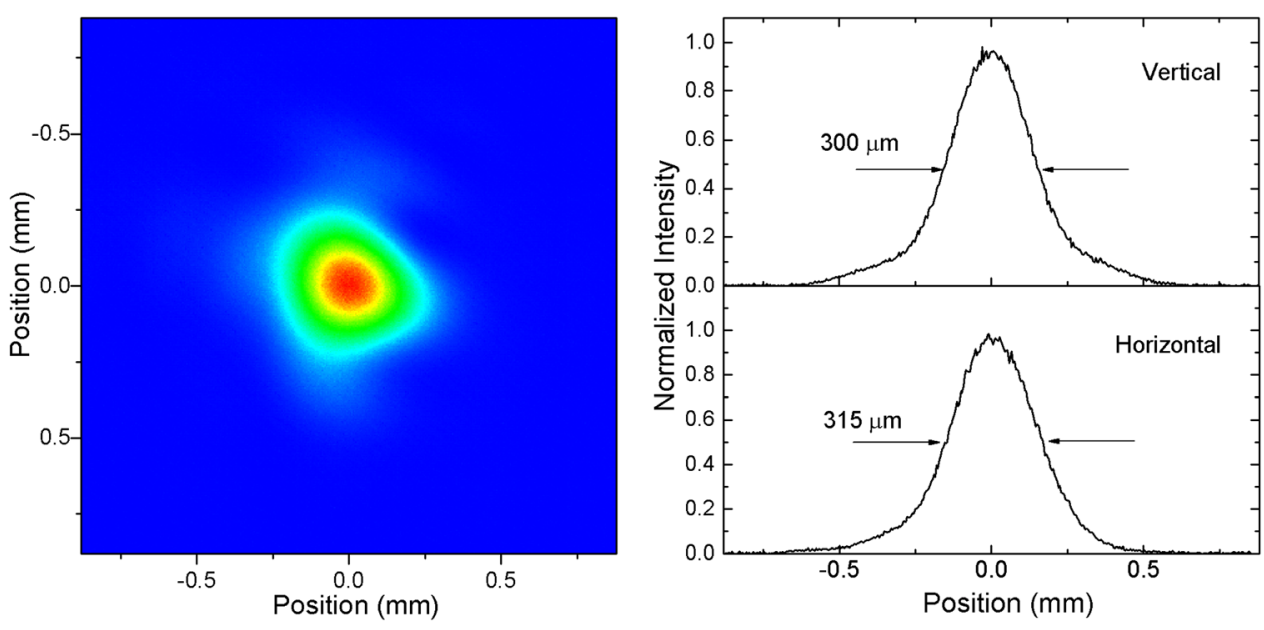

FIG. 4. Left: Intensity profile measurement at focus of $\mathrm{f}=6.5 \mathrm{~m}$ mirror with a 12-bit CCD camera; right: horizontal and vertical line-out (diameter is $310 \mu \mathrm{m}$ at half-maximum). 


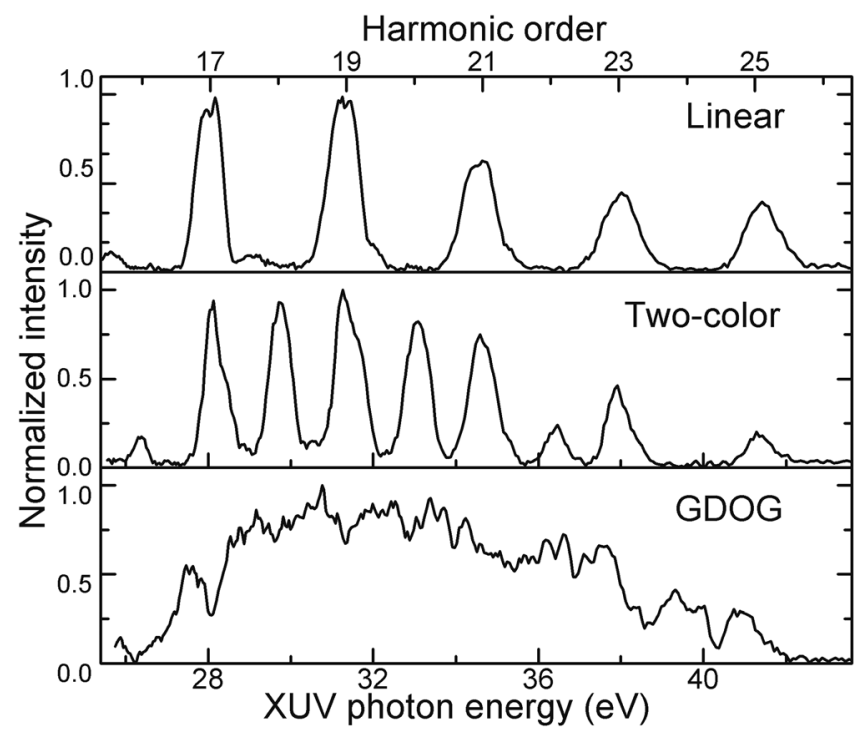

FIG. 5. Single-shot XUV spectra generated in argon under different gating conditions.

achieve high energy without incurring substantial gain narrowing. This is also the first demonstration of using multi-cycle laser pulses at the $10 \mathrm{TW}$ level with the GDOG technique to generate an XUV continuum. Our preliminary results pave the way for scaling up XUV photon flux by further increasing the power of the driving laser.

This material is based upon work supported by the U.S. Army Research Office and the National Science Foundation under Grant Number 1068604. Huaping Zang and Xiaowei Wang recognize the support from the China Scholarship Council.

${ }^{1}$ F. Krausz and M. Ivanov, Rev. Mod. Phys. 81, 163 (2009).

${ }^{2}$ Z. Chang and P. Corkum, J. Opt. Soc. Am. B 27, 9 (2010).

${ }^{3}$ F. Ferrari, F. Calegari, M. Lucchini, C. Vozzi, S. Stagira, G. Sansone, and M. Nisoli, Nature Photon. 4, 875 (2010).

${ }^{4}$ X. Feng, S. Gilbertson, H. Mashiko, H. Wang, S. D. Khan, M. Chini, Y. Wu, K. Zhao, and Z. Chang, Phys. Rev. Lett. 103, 183901 (2009).

${ }^{5}$ J. Seres, A. Müller, E. Seres, K. O'Keeffe, M. Lenner, R. Herzog, D. Kaplan, C. Spielmann, and F. Krausz, Opt. Lett. 28, 1832 (2003).

${ }^{6}$ H. Takada and K. Torizuka, IEEE J. Quantum Electron. 12, 201 (2006).

${ }^{7}$ A. Amani Eilanlou, Y. Nabekawa, K. L. Ishikawa, H. Takahashi, and K. Midorikawa, Opt. Express 16, 13431 (2008).

${ }^{8}$ K. Ertel, C. Hooker, S. J. Hawkes, B. T. Parry, and J. L. Collier, Opt. Express 16, 8039 (2008).

${ }^{9}$ K.-H. Hong and C. H. Nam, Jpn. J. Appl. Phys. Part 1 43, 5289 (2004).

${ }^{10}$ E. Takahashi, Y. Nabekawa, T. Otsuka, M. Obara, and K. Midorikawa, Phys. Rev. A 66, 021802 (2002).

${ }^{11}$ E. J. Takahashi, Y. Nabekawa, H. Mashiko, H. Hasegawa, A. Suda, and K. Midorikawa, IEEE J. Quantum Electron. 10, 1315 (2004).

${ }^{12}$ X. Wang, M. Chini, Y. Cheng, Y. Wu, and Z. Chang, Appl. Opt. 52, 323 (2013).

${ }^{13}$ K. Zhao, Q. Zhang, M. Chini, Y. Wu, X. Wang, and Z. Chang, Opt. Lett. 37, 3891 (2012). 